[Article]

\title{
稀土掺杂对氯化氢氧化制氯气 $\mathrm{CuO}-\mathrm{CeO}_{2}-\mathrm{SiO}_{2}$ 催化剂 结构和性能的影响
}

\author{
谢兴星 ${ }^{1,2}$ \\ 费兆阳 ${ }^{1,2, *}$ \\ 邹 冲 ${ }^{1,2}$ \\ 李郑州 ${ }^{1,2}$ \\ 陈 献 $^{2}$ \\ 汤吉海 $^{2}$ \\ 崔咪芬 $^{2}$ \\ 乔旭 ${ }^{1,2, *}$ \\ ('南京工业大学材料化学工程国家重点实验室, 南京 210009; 2南京工业大学化学化工学院, 南京 210009)
}

\begin{abstract}
摘要: 采用模板法制备了 $\mathrm{CuO}-\mathrm{CeO}_{2}-\mathrm{SiO}_{2}$ 和稀土掺杂的 $\mathrm{CuO}-\mathrm{Ce}_{0.9} \mathrm{M}_{0.1} \mathrm{O}_{2}-\mathrm{SiO}_{2}(\mathrm{M}=\mathrm{La}, \mathrm{Pr}, \mathrm{Nd}$ )催化剂. 运用X 射线衍射(XRD), $N_{2}$ 吸附-脱附, 透射电镜(TEM), 拉曼(Raman)光谱, $X$ 射线光电子能谱(XPS)和氢气-程序升温 还原 $\left(H_{2}-T P R\right)$ 等手段对催化剂的结构进行表征, 并考察稀土掺杂对氯化氢催化氧化制氯气性能的影响. 结果表 明, 稀土掺杂进入 $\mathrm{CeO}_{2}$ 晶格中形成良好的固溶体结构, 获得更小的晶粒尺寸和更高的比表面积, 并且显著提高 了固溶体的表面氧空位浓度. 稀土掺杂显著影响了催化剂的氯化氢催化氧化活性, 活性顺序为: CuO$\mathrm{Ce}_{0.9} \mathrm{La}_{0.1} \mathrm{O}_{2}-\mathrm{SiO}_{2}>\mathrm{CuO}-\mathrm{Ce}_{0.9} \mathrm{Nd}_{0.1} \mathrm{O}_{2}-\mathrm{SiO}_{2}>\mathrm{CuO}-\mathrm{Ce}_{0.9} \mathrm{Pr}_{0.1} \mathrm{O}_{2}-\mathrm{SiO}_{2}>\mathrm{CuO}-\mathrm{CeO}_{2}-\mathrm{SiO}_{2}$, 固溶体氧空位浓度的高低

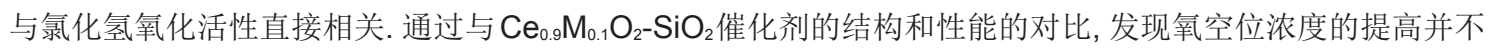
能增强在固溶体表面发生的氯化氢氧化反应. 动力学测试显示, 稀土掺杂后, 氧分子的吸附成为反应过程的决 速步骤. 但在 $V\left(\mathrm{O}_{2}\right): V(\mathrm{HCl})=1$ 条件下, 更高的氧空位浓度导致了固溶体更低的氯化氢氧化反应速率. 结合机理 分析认为, $\mathrm{CuO}-\mathrm{Ce}_{0.9} \mathrm{M}_{0.1} \mathrm{O}_{2}-\mathrm{SiO}_{2}$ 催化剂更高的氧空位浓度增强了固溶体表面的“氧溢流”, 加快了氯化氢氧化 的整体反应速率, 这是 $\mathrm{CuO}-\mathrm{Ce}_{0.9} \mathrm{M}_{0.1} \mathrm{O}_{2}-\mathrm{SiO}_{2}$ 具备高活性的关键.
\end{abstract}

关键词：稀土；钥铜复合氧化物；氯化氢；催化氧化；氯气；氧空位

中图分类号: 0643

\section{Effects of Rare-Earth Additives on Structures and Performances of $\mathrm{CuO}-\mathrm{CeO}_{2}-\mathrm{SiO}_{2}$ Catalysts for Recycling $\mathrm{Cl}_{2}$ from $\mathrm{HCl}$ Oxidation}

\author{
XIE Xing-Xing ${ }^{1,2}$ \\ FEI Zhao-Yang ${ }^{1,2, *}$ \\ ZOU Chong ${ }^{1,2}$ \\ LI Zheng-Zhou ${ }^{1,2}$ \\ CHEN Xian ${ }^{2}$ \\ TANG Ji-Hai ${ }^{2}$ \\ CUI Mi-Fen ${ }^{2}$ \\ QIAO Xu $\mathrm{u}^{1,2, *}$ \\ ('State Key Laboratory of Materials-Oriented Chemical Engineering, Nanjing Tech University, Nanjing 210009, P. R. China; \\ ${ }^{2}$ College of Chemistry and Chemical Engineering, Nanjing Tech University, Nanjing 210009, P. R. China)
}

\begin{abstract}
CuO}-\mathrm{CeO}_{2}-\mathrm{SiO}_{2}$ and rare-earth-doped $\mathrm{CuO}-\mathrm{Ce}_{0.9} \mathrm{M}_{0.1} \mathrm{O}_{2}-\mathrm{SiO}_{2}(\mathrm{M}=\mathrm{La}, \mathrm{Pr}, \mathrm{Nd})$ catalysts for recycling $\mathrm{Cl}_{2}$ from $\mathrm{HCl}$ oxidation were prepared by a template method, using activated carbon as a hard template. The catalyst structures were determined using X-ray diffraction (XRD), $\mathrm{N}_{2}$ adsorption-desorption, transmission electron microscopy (TEM), Raman spectroscopy, X-ray photoelectron spectroscopy (XPS), and $\mathrm{H}_{2}$ temperatureprogrammed reduction $\left(\mathrm{H}_{2}\right.$-TPR). The catalytic performances were also investigated. The results showed that
\end{abstract}

Received: December 23, 2014; Revised: April 14, 2015; Published on Web: April 14, 2015.

"Corresponding authors. FEI Zhao-Yang, Email: zhaoyangfei@njtech.edu.cn; Tel: +86-25-83587168. QIAO Xu, Email: qct@njtech.edu.cn; Tel: $+86-25-83172298$.

The project was supported by the National Key Technology Research and Development Program of the Ministry of Science and Technology of China (2011BAE18B01), Research and Development Program of Jiangsu Province, China (BE2011830), Higher Education Natural Science Foundation of Jiangsu Province, China (13KJB530006), National Natural Science Foundation of China (21306089) and China Postdoctoral Science Foundation (2013M531340). 国家科技支撑计划(2011BAE18B01), 江苏省科技支撑计划(BE2011830), 江苏省高校自然科学基金面上项目(13KJB530006), 国家自然科学基 金(21306089)和中国博士后基金(2013M531340)资助

(c) Editorial office of Acta Physico-Chimica Sinica 
$\mathrm{La}, \mathrm{Pr}$, and $\mathrm{Nd}$ cations were incorporated into the $\mathrm{CeO}_{2}$ lattice and formed nanosized solid solutions; this greatly reduced the catalyst grain sizes, leading to higher surface areas. In addition, the oxygen vacancy concentrations were significantly improved. The changes in the structures and surface properties of the solid solutions significantly affected the $\mathrm{HCl}$ catalytic oxidation performances. The order of the activities of various catalysts was $\mathrm{CuO}-\mathrm{Ce}_{0.9} \mathrm{Laa}_{0.1} \mathrm{O}_{2}-\mathrm{SiO}_{2}>\mathrm{CuO}-\mathrm{Ce}_{0.9} \mathrm{Nd}_{0.1} \mathrm{O}_{2}-\mathrm{SiO}_{2}>\mathrm{CuO}-\mathrm{Ce}_{0.9} \mathrm{Pr}_{0.1} \mathrm{O}_{2}-\mathrm{SiO}_{2}>\mathrm{CuO}-\mathrm{CeO}_{2}-\mathrm{SiO}_{2}$. The oxygen vacancy concentrations of the solid solutions were strongly related to their catalytic activities. However, the structures and performances of the $\mathrm{Ce}_{0.9} \mathrm{M}_{0.1} \mathrm{O}_{2}-\mathrm{SiO}_{2}$ catalysts showed that an increase in the number of oxygen vacancies resulted in decreased catalytic activities of the solid solutions. Kinetic studies showed that oxygen adsorption could be the rate-determining step for rare-earth-doped catalysts; a higher oxygen vacancy concentration in the solid solution led to a slower reaction rate when the volumetric flow ratio of $\mathrm{O}_{2}$ to $\mathrm{HCl}$ was 1 . For the $\mathrm{CuO}-$ $\mathrm{Ce}_{0.9} \mathrm{M}_{0.1} \mathrm{O}_{2}-\mathrm{SiO}_{2}$ catalysts, spillover of oxygen species in the solid solution into the highly dispersed $\mathrm{CuO}$ interfaces was enhanced, which increased the overall reaction rate and gave high activity.

Key Words: Rare earth; $\mathrm{CuO}-\mathrm{CeO}_{2} ; \quad \mathrm{HCl}$; Catalytic oxidation; Chlorine; Oxygen vacancy

\section{1 引言}

氯气作为一种重要的基础化工原料, 广泛应用 于造纸、医药、纺织、石油化工、环保等行业. 但是, 以氯气为原料的许多生产过程会副产大量的氯化 氢, 以快速发展的聚氨酯和聚碳酸酯行业为例, 全 部氯元素都是以副产氯化氢的形式排出. ${ }^{1}$ 副产氯化 氢如果不能被合理的利用, 不仅会造成氯资源的极 大浪费, 同时还会对环境造成严重的污染. 氯化氢 通过催化氧化法制氯气, 能够实现氯资源的循环利 用, 是具有广阔应用前景的绿色环保工艺. ${ }^{2-4}$

目前, 住友和拜耳公司分别开发了 $\mathrm{RuO}_{2}$ 催化 剂, 其具备较好的低温活性和催化稳定性, 成功实 现了催化氯化氢氧化制氯气的工业应用. ${ }^{5,6}$ 但是, $\mathrm{RuO}_{2}$ 催化剂存在成本昂贵, 且活性温区窄, 高温易 流失等问题, 限制了其进一步大规模使用. ${ }^{7}$ 因此, 成 本较低且具备高活性和稳定性的催化剂的开发已 成为氯化氢氧化研究的热点. $\mathrm{CeO}_{2}$ 及铈基复合氧化 物因其优异的储释氧性能而被广泛应用. ${ }^{8-10}$ 最近研 究显示 $\mathrm{CeO}_{2}$ 在较宽温区范围内具有较好的氯化氢 氧化催化性能, 被认为是可替代 $\mathrm{RuO}_{2}$ 的新型氯化氢 氧化催化剂. ${ }^{11}$ 以 $\mathrm{ZrO}_{2}$ 为载体采用浸渍法制备了负 载型 $\mathrm{CeO}_{2} / \mathrm{ZrO}_{2}$ 催化剂, $\mathrm{CeO}_{2}$ 高度分散在 $\mathrm{ZrO}_{2}$ 的表 面形成了纳米薄层, 显示出良好的氧化还原能力和 抗氯化性能. ${ }^{12}$ 铜铁矿结构的 $\mathrm{CuCrO}_{2}$ 与 $\mathrm{CeO}_{2}$ 复合后 氯化氢氧化催化活性较单组分提高四倍, 而 $\mathrm{CeO}_{2}$ 的 氧空位的存在显著增强了其催化性能. ${ }^{13}$ 在前期的 工作中, ${ }^{14}$ 我们采用 $\mathrm{Y}$ 分子篮为载体, 制备出的 $\mathrm{CuO}-$ $\mathrm{CeO}_{2} / \mathrm{Y}$ 催化剂在氯化氢氧化反应中表现出极高的 活性和稳定性, 具备良好的工业应用前景. 详细的 研究表明, ${ }^{15}$ 高分散的 $\mathrm{CuO}$ 为最主要的活性组分,
$\mathrm{CuO}$ 和 $\mathrm{CeO}_{2}$ 之间存在着协同作用, 铈铜固溶体的表 面氧空位能够促进氧分子在催化剂表面的吸附和 活化, 这是催化剂具备高活性的关键.

稀土的掺杂可以有效改变 $\mathrm{CeO}_{2}$ 的结构和物理 化学性质, 尤其是表面氧空位浓度和氧化还原性 能. ${ }^{16,17}$ 然而, 对于稀土掺杂对氯化氢氧化 $\mathrm{CuO}-\mathrm{CeO}_{2}$ 催化剂结构和性能影响的研究甚少. 本文采用模板 法制备了 $\mathrm{CuO}-\mathrm{CeO}_{2}-\mathrm{SiO}_{2}$ 和 $\mathrm{CuO}-\mathrm{Ce}_{0.9} \mathrm{M}_{0.1} \mathrm{O}_{2}-\mathrm{SiO}_{2}(\mathrm{M}=$ $\mathrm{La}, \mathrm{Pr}, \mathrm{Nd}$ )催化剂, 通过多种表征手段考察稀土掺 杂对催化剂的结构以及氯化氢氧化性能影响. 同时 还制备了 $\mathrm{Ce}_{0.9} \mathrm{M}_{0.1} \mathrm{O}_{2}-\mathrm{SiO}_{2}(\mathrm{M}=\mathrm{La}, \mathrm{Pr}, \mathrm{Nd})$ 催化剂, 通 过与其结构和性能的对比, 并结合动力学手段, 尝 试阐述氯化氢氧化在 $\mathrm{CuO}-\mathrm{Ce}_{0.9} \mathrm{M}_{0.1} \mathrm{O}_{2}-\mathrm{SiO}_{2}$ 表面反应 过程.

\section{2 实验部分}

\section{1 催化剂制备}

$\mathrm{CuO}-\mathrm{CeO}_{2}-\mathrm{SiO}_{2}$ 催化剂采用模板法制备, 合成 方法和条件如下: 取原硅酸四乙酯(TEOS) $1.50 \mathrm{~g}$ (国 药集团化学试剂有限公司, 分析纯), 乙醇 $1.82 \mathrm{~g}$ (无 锡亚盛化工有限公司, 分析纯), 加入到 $6 \mathrm{~g}$ 活性炭中 摚拌均匀(江苏竹溪活性炭有限公司, 20-50目, 分析纯), 于管式炉 $350{ }^{\circ} \mathrm{C}$ 焙烧 $0.5 \mathrm{~h}$ 得到 “ $\mathrm{SiO}_{2}$-活 性炭” 混合物. 将 $0.30 \mathrm{~g} \mathrm{Cu}\left(\mathrm{NO}_{3}\right)_{2} \cdot 3 \mathrm{H}_{2} \mathrm{O}$ 和 $6.2 \mathrm{~g}$ $\mathrm{Ce}\left(\mathrm{NO}_{3}\right)_{3} \cdot 6 \mathrm{H}_{2} \mathrm{O}$ (国药集团化学试剂有限公司, 分析 纯)溶于 $3.8 \mathrm{~mL}$ 去离子水中配成溶液, 然后在搅拌 下将该溶液加入到上述的“ $\mathrm{SiO}_{2}$-活性炭” 混合物中, 摚拌均匀后于管式炉 $550{ }^{\circ} \mathrm{C}$ 焙烧 $3 \mathrm{~h}$, 即制得 $\mathrm{CuO}-$ $\mathrm{CeO}_{2}-\mathrm{SiO}_{2}$ 催化剂. 采用相同的制备方法, 将上述 体系中一部分 $\mathrm{Ce}\left(\mathrm{NO}_{3}\right)_{3} \cdot 6 \mathrm{H}_{2} \mathrm{O}$ 用 $\mathrm{La}\left(\mathrm{NO}_{3}\right)_{3} \cdot 6 \mathrm{H}_{2} \mathrm{O}$ 、 
$\operatorname{Pr}\left(\mathrm{NO}_{3}\right)_{3} \cdot 6 \mathrm{H}_{2} \mathrm{O}$ 或者 $\mathrm{Nd}\left(\mathrm{NO}_{3}\right)_{3} \cdot 6 \mathrm{H}_{2} \mathrm{O}$ (国药集团化学 试剂有限公司, 分析纯)替换, 使体系中的铈离子与 掺杂稀土金属离子的摩尔比为 9:1, 制备出的催化剂 记为 $\mathrm{CuO}-\mathrm{Ce}_{0.9} \mathrm{M}_{0.1} \mathrm{O}_{2}-\mathrm{SiO}_{2}(\mathrm{M}=\mathrm{La}, \mathrm{Pr}, \mathrm{Nd})$.

\section{2 催化剂表征}

$\mathrm{XRD}$ 在日本 Rigaku 公司 SarmtLab 衍射仪上进 行检测, 采用 $\mathrm{Cu} K_{a}$ 为射线源 $(\lambda=0.15406 \mathrm{~nm})$, 激发 电压为 $40 \mathrm{kV}$, 电流 $100 \mathrm{~mA}$, 扫描速率 $20\left(^{\circ}\right) \cdot \mathrm{min}^{-1}$, 扫描范围 $2 \theta=10^{\circ}-80^{\circ}$. 比表面积(BET)测试在日本 贝尔公司 BELSORP II 型吸附仪上进行采用 $\mathrm{N}_{2}$ 吸 附法进行, 样品在 $200{ }^{\circ} \mathrm{C}$ 下真空预处理 $3 \mathrm{~h}$, 然后 在 $-196{ }^{\circ} \mathrm{C}$ 下进行吸脱附实验. TEM 是在日本 JEM2100 型投射电子显微镜上进行测试的. Raman 光谱 是在 Confocal Renishaw 公司的 RM-1000 型表面增 强激光拉曼光谱仪上进行测试, 激光光源采用 $\mathrm{He}$ Cd 光源器, 波长为 $514 \mathrm{~nm}$, 扫描范围 100-2000 $\mathrm{cm}^{-1}$, 将在每个样品表面的三个不同位置重复扫描三次 后的结果进行重合, 对照标准样品的激光拉曼光谱 (LRS) 谱进行峰的指认. XPS 实验使用 Thermo ESCALAB 250 型 X 射线光电子能谱仪测试, 实验中使 用 $\mathrm{Al} K_{a}$ 光源 $(1486.6 \mathrm{eV})$, 能量分辨率 $20 \mathrm{eV}$, 实验数 据以 $\mathrm{C} 1 s$ 谱的标准结合能位置 $(284.6 \mathrm{eV}$ )为基准校 准. $\mathrm{H}_{2}$-TPR 实验在美国麦克公司 AutoChem II 2920 上进行, 称取 $50 \mathrm{mg}$ 样品置于石英反应管中, 通入高 纯 $\mathrm{Ar}$ 并升温至 $200^{\circ} \mathrm{C}$ 预处理 $1 \mathrm{~h}$, 冷却至 $50^{\circ} \mathrm{C}$, 然后 再通入 $\mathrm{H}_{2}(10 \%)-\operatorname{Ar}(90 \%)$ 的还原气, 开始程序升温 还原至 $900{ }^{\circ} \mathrm{C}$, 气体流速为 $50 \mathrm{~mL} \cdot \mathrm{min}^{-1}$, 升温速率 为 $10^{\circ} \mathrm{C} \cdot \mathrm{min}^{-1}$, 采用热导检测器 $(\mathrm{TCD})$ 检测.

\section{3 催化剂的活性评价}

催化剂活性评价是在固定床管式反应器中进 行, 反应管内径为 $24 \mathrm{~mm}$, 热电偶套管外径为 $4 \mathrm{~mm}$, 催化剂装填量为 $5 \mathrm{~g}$, 粒径为 0.45-1.25 mm (16-40 目), 用十倍石英砂对催化剂进行稀释, 将其装填 于石英反应器中部. 反应温度为 $350-410{ }^{\circ} \mathrm{C}$, 反应 气体为 $\mathrm{HCl}$ 和 $\mathrm{O}_{2}, \mathrm{HCl}$ 流量为 $80 \mathrm{~mL} \cdot \mathrm{min}^{-1}, V\left(\mathrm{O}_{2}\right)$ : $V(\mathrm{HCl})=1$, 反应产物用 $\mathrm{KI}$ 溶液进行吸收后, 用碘量 法及酸碱滴定法测定生成的氯气及未反应的氯化 氢, 计算出氯化氢的转化率 $\left(X_{\mathrm{HC}}\right)$ :

$$
X_{\mathrm{HCl}}=\frac{C_{\mathrm{Na}_{2} \mathrm{~S}_{2} \mathrm{O}_{3}} V_{\mathrm{Na}_{2} \mathrm{~S}_{2} \mathrm{O}_{3}}}{C_{\mathrm{Na}_{2} \mathrm{~S}_{2} \mathrm{O}_{3}} V_{\mathrm{Na}_{2} \mathrm{~S}_{2} \mathrm{O}_{3}}+C_{\mathrm{NaOH}_{3}} V_{\mathrm{NaOH}}} \times 100 \%
$$

\section{3 结果与讨论}

\section{$3.1 \mathrm{CuO}-\mathrm{Ce}_{0.9} \mathrm{M}_{0.1} \mathrm{O}_{2}-\mathrm{SiO}_{2}$ 表征}

\subsubsection{XRD表征}

$\mathrm{CuO}-\mathrm{Ce}_{0.9} \mathrm{M}_{0.1} \mathrm{O}_{2}-\mathrm{SiO}_{2}$ 催化剂的 XRD 谱图如图 1 所示. 可以看到, $\mathrm{CuO}-\mathrm{CeO}_{2}-\mathrm{SiO}_{2}$ 的衍射图谱出现对 应于 $\mathrm{CeO}_{2}$ 立方萤石结构的特征衍射峰; 在 $15^{\circ}-20^{\circ}$ 出现对应于 $\mathrm{SiO}_{2}$ 短程有序、长程无序的无定型形式 的特征衍射峰, ${ }^{18}$ 未观察到对应于晶相 $\mathrm{CuO}$ 的特征 衍射峰, 这表明 $\mathrm{Cu}$ 物种可能以氧化物形式在 $\mathrm{CeO}_{2}-$ $\mathrm{SiO}_{2}$ 表面, 高度分散或者部分可能掺入 $\mathrm{CeO}_{2}$ 的晶格 中. 从 $\mathrm{CuO}-\mathrm{Ce}_{0.9} \mathrm{M}_{0.1} \mathrm{O}_{2}-\mathrm{SiO}_{2}$ 的衍射图谱看到对应于 无定型 $\mathrm{SiO}_{2}$ 的特征衍射峰出现偏移, 这表明稀土掺 杂后导致 $\mathrm{SiO}_{2}$ 短程结构发生了一定的改变. 此外, 只观察到对应于 $\mathrm{CeO}_{2}$ 立方萤石结构的特征衍射峰, 而特征峰均向低角度偏移, 这表明掺杂的稀土金属 进入了 $\mathrm{CeO}_{2}$ 的晶格中并形成了固溶体结构. ${ }^{16}$ 根据 Bragg 公式计算的各催化剂的晶胞参数列于表 1. 从 表 1 可以看到, 稀土掺杂后的催化剂晶胞参数均变 大, 这是因为离子半径比 $\mathrm{Ce}^{4+}(0.097 \mathrm{~nm})$ 更大的 $\mathrm{Pr}^{3+}$ $(0.113 \mathrm{~nm}), \mathrm{Nd}^{3+}(0.111 \mathrm{~nm})$ 和 $\mathrm{La}^{3+}(0.110 \mathrm{~nm})$ 进入了 $\mathrm{CeO}_{2}$ 的晶格中, 导致了晶胞的扩张. 此外, 由 Scherrer 公式计算的各催化剂的平均晶粒尺寸列于表 1 . 可 以看到, 采用模板法制备的 $\mathrm{CuO}-\mathrm{CeO}_{2}-\mathrm{SiO}_{2}$ 晶粒尺 寸为 $5.1 \mathrm{~nm}$, 稀土掺杂后可以获得更小的晶粒尺寸. 其中, $\mathrm{CuO}-\mathrm{Ce}_{0.9} \mathrm{La}_{0.1} \mathrm{O}_{2}-\mathrm{SiO}_{2}$ 平均晶粒尺寸最小仅为 $4.1 \mathrm{~nm}$.

\subsection{2 $\mathrm{N}_{2}$ 吸附-脱附测试}

图 2 是 $\mathrm{CuO}-\mathrm{Ce}_{0.9} \mathrm{M}_{0.1} \mathrm{O}_{2}-\mathrm{SiO}_{2}$ 催化剂的 $\mathrm{N}_{2}$ 吸脱附 等温线. 从图中可以看出催化剂的 $\mathrm{N}_{2}$ 吸脱-脱附等 温线属于 IV 型等温线, 表明所有样品均具有介孔结 构. 此外, H3 型回滞环的存在表明这些催化剂的孔 道并不规则, 它们具有主要为堆积孔构成的混合孔

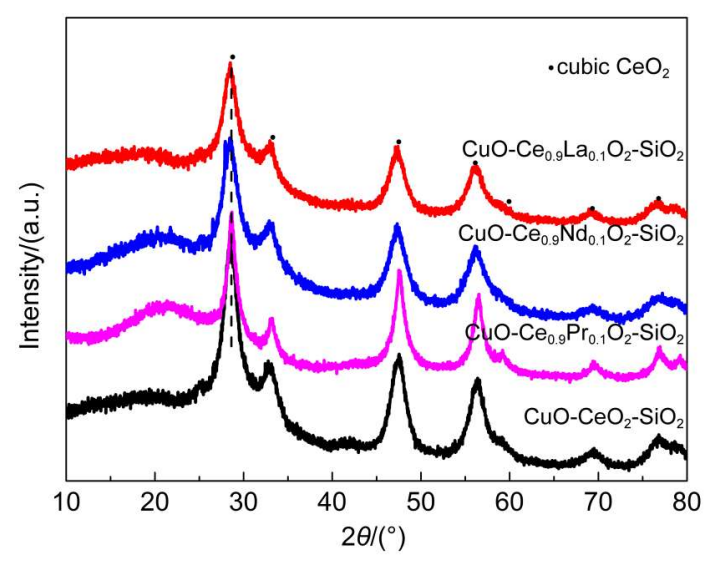

图 $1 \mathrm{CuO}-\mathrm{Ce}_{0.9} \mathrm{M}_{0.1} \mathrm{O}_{2}-\mathrm{SiO}_{2}$ 催化剂的XRD 谱图

Fig.1 XRD patterns of $\mathrm{CuO}-\mathrm{Ce}_{0.9} \mathrm{M}_{0.1} \mathrm{O}_{2}-\mathrm{SiO}_{2}$ catalysts 
表 $1 \mathrm{CuO}-\mathrm{CeO}_{2}-\mathrm{SiO}_{2}$ 和 $\mathrm{CuO}-\mathrm{Ce}_{0.9} \mathrm{M}_{0.1} \mathrm{O}_{2}-\mathrm{SiO}_{2}$ 催化剂的物理化学性质

Table 1 Physical chemical properties of $\mathrm{CuO}-\mathrm{CeO}_{2}-\mathrm{SiO}_{2}$ and $\mathrm{CuO}-\mathrm{Ce}_{0.9} \mathrm{M}_{0.1} \mathrm{O}_{2}-\mathrm{SiO}_{2}$ catalysts

\begin{tabular}{|c|c|c|c|c|c|c|c|c|c|}
\hline Catalyst & Cell parameter/nm & Crystal size $/ \mathrm{nm}^{\text {a }} S$ & $S_{\mathrm{BET}} /\left(\mathrm{m}^{2} \cdot \mathrm{g}^{-1}\right)^{\mathrm{b}}$ & $V_{\mathrm{p}} /\left(\mathrm{cm}^{3} \cdot \mathrm{g}^{-1}\right)^{\mathrm{b}}$ & $\left(A_{605} / A_{459}\right) / \%{ }^{\mathrm{c}}$ & $\begin{array}{l}\text { Consumption amount } \\
\text { of } \mathrm{H}_{2} /\left(\mathrm{cm}^{3} \cdot \mathrm{g}^{-1}\right)^{\mathrm{d}}\end{array}$ & $X_{\mathrm{Cu}^{2+}} / \%{ }^{\mathrm{e}}$ & $X_{\mathrm{M}^{3+}} / \% \%^{\mathrm{f}}$ & $X_{\mathrm{Ce}^{3+}} / \% \mathrm{o}^{\mathrm{g}}$ \\
\hline $\mathrm{CuO}-\mathrm{CeO}_{2}-\mathrm{SiO}_{2}$ & 0.0538 & 5.1 & 127.7 & 0.2450 & 9.54 & 34.96 & 29.9 & - & 11.59 \\
\hline $\mathrm{CuO}-\mathrm{Ce}_{0.9} \mathrm{Pr}_{0.1} \mathrm{O}_{2}-\mathrm{SiO}_{2}$ & 0.0540 & 5.0 & 128.5 & 0.2157 & 26.80 & 35.10 & 30.3 & 9.1 & 11.74 \\
\hline $\mathrm{CuO}-\mathrm{Ce}_{0.9} \mathrm{Nd}_{0.1} \mathrm{O}_{2}-\mathrm{SiO}_{2}$ & 0.0541 & 4.4 & 139.0 & 0.2576 & 29.80 & 36.13 & 30.3 & 8.5 & 12.01 \\
\hline $\mathrm{CuO}-\mathrm{Ce}_{0.9} \mathrm{La}_{0.1} \mathrm{O}_{2}-\mathrm{SiO}_{2}$ & 0.0541 & 4.1 & 151.9 & 0.2597 & 41.43 & 41.92 & 31.8 & 8.6 & 14.76 \\
\hline
\end{tabular}

a calculation from the peak during $28.4^{\circ}$ in the XRD pattern using the Scherrer equation. ${ }^{\mathrm{b}}$ surface area derived from BET method.

${ }^{\mathrm{c}} A_{605} / A_{459}$ calculated from Raman results. ${ }^{\mathrm{d}}$ calculation from the peak at $210{ }^{\circ} \mathrm{C}$ in $\mathrm{H}_{2}$-TPR profiles. ${ }^{\mathrm{e}}$ surface atomic ratio of

$\mathrm{Cu} 2 p /(\mathrm{Ce} 3 d+\mathrm{M} 3 d)$ determined by XPS, M= La, Pr, Nd. ${ }^{\mathrm{f}}$ Surface atomic ratio of M $3 d /(\mathrm{Ce} 3 d+\mathrm{M} 3 d)$ determined by XPS,

${ }^{g}$ surface atomic ratio of $\mathrm{Ce}^{3+} /\left(\mathrm{Ce}^{3+}+\mathrm{Ce}^{4+}\right)$ determined by XPS

道结构. $\mathrm{CuO}-\mathrm{Ce}_{0.9} \mathrm{M}_{0.1} \mathrm{O}_{2}-\mathrm{SiO}_{2}$ 催化剂的比表面积和 孔容数据列于表 1 中, 可见各催化剂孔容比较接近, 为 $0.2157-0.2597 \mathrm{~m}^{3} \cdot \mathrm{g}^{-1}$. 而 $\mathrm{CuO}-\mathrm{Ce}_{0.9} \mathrm{M}_{0.1} \mathrm{O}_{2}-\mathrm{SiO}_{2}$ 催 化剂均具有较高比表面积, $\mathrm{CuO}-\mathrm{CeO}_{2}-\mathrm{SiO}_{2}$ 为 127.7 $\mathrm{m}^{2} \cdot \mathrm{g}^{-1}$, 稀土掺杂后催化剂比表面积有所提高, 这主 要归因于稀土的掺入固溶体抑制了其个体在焙烧 过程中的结晶, ${ }^{19}$ 从而导致了较小的晶粒尺寸, 显示 出更大的比表面积. 其中, $\mathrm{CuO}-\mathrm{Ce}_{0.9} \mathrm{La}_{0.1} \mathrm{O}_{2}-\mathrm{SiO}_{2}$ 比表 面积显著提高至 $151.9 \mathrm{~m}^{2} \cdot \mathrm{g}^{-1}$.

\subsubsection{TEM 结果}

CuO- $\mathrm{Ce}_{0.9} \mathrm{M}_{0.1} \mathrm{O}_{2}-\mathrm{SiO}_{2}$ 催化剂的 TEM 图见图3. 可以看到, $\mathrm{CuO}-\mathrm{CeO}_{2}-\mathrm{SiO}_{2}$ 催化剂的晶粒尺寸由 6$8 \mathrm{~nm}$ 左右的颗粒不规则排列而成, 稀土掺杂后催化 剂晶粒尺寸更小, 粒径为 $6 \mathrm{~nm}$ 以下. 从图 3 中选区 电子衍射图可以清晰观测到的 $\mathrm{Pr} 、 \mathrm{Nd}$ 和 $\mathrm{La}$ 催化剂 的 $\mathrm{CeO}_{2}$ 颗粒的(111)面的晶格条纹, 测量的晶面间距 为 $0.320 \mathrm{~nm}$, 掺杂 $\mathrm{Pr} 、 \mathrm{Nd}$ 和 $\mathrm{La}$ 后(111)面的晶面间距 分别增至 $0.322 、 0.322$ 和 $0.326 \mathrm{~nm}$, 这表明稀土掺杂 进入 $\mathrm{CeO}_{2}$ 的晶格并导致了晶胞扩张, 这与 $\mathrm{XRD}$ 结 果相一致. 此外, 对 $\mathrm{CuO}-\mathrm{Ce}_{0.9} \mathrm{M}_{0.1} \mathrm{O}_{2}-\mathrm{SiO}_{2}$ 催化剂任选

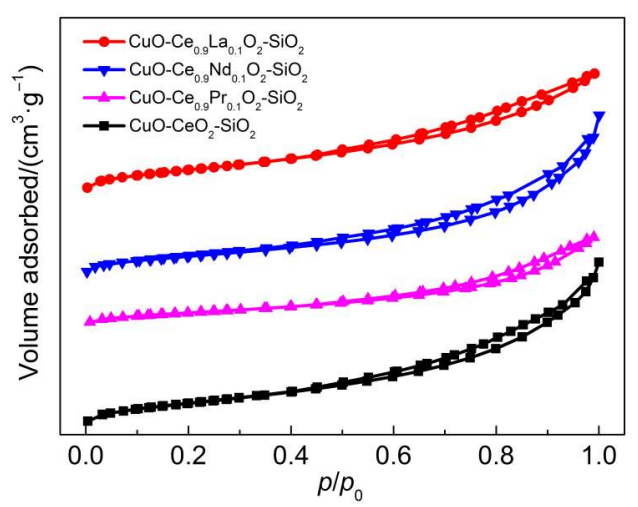

图 $2 \mathrm{CuO}-\mathrm{Ce}_{0.9} \mathrm{M}_{0.1} \mathrm{O}_{2}-\mathrm{SiO}_{2}$ 催化剂的 $\mathrm{N}_{2}$ 吸脱附等温线

Fig.2 Isotherm of $\mathrm{N}_{2}$ adsorption-desorption for $\mathrm{CuO}-\mathrm{Ce}_{0.9} \mathrm{M}_{0.1} \mathrm{O}_{2}-\mathrm{SiO}_{2}$ catalysts
区域的元素的含量进行检测, 如图 4所示. 可以看到 在不同区域, 稀土金属 $\mathrm{Pr} 、 \mathrm{Nd}$ 和 $\mathrm{La}$ 分别在 $\mathrm{CuO}-$ $\mathrm{Ce}_{0.9} \mathrm{La}_{0.1} \mathrm{O}_{2}-\mathrm{SiO}_{2} 、 \mathrm{CuO}-\mathrm{Ce}_{0.9} \mathrm{Nd}_{0.1} \mathrm{O}_{2}-\mathrm{SiO}_{2}$ 和 $\mathrm{CuO}-$ $\mathrm{Ce}_{0.9} \mathrm{Pr}_{0.1} \mathrm{O}_{2}-\mathrm{SiO}_{2}$ 催化剂的表面区域的分散是较为均 匀的.

\subsubsection{Raman 表征}

$\mathrm{CuO}-\mathrm{Ce}_{0.9} \mathrm{M}_{0.1} \mathrm{O}_{2}-\mathrm{SiO}_{2}$ 催化剂的 Raman 谱图如图 5 所示. $\mathrm{CuO}-\mathrm{CeO}_{2}-\mathrm{SiO}_{2}$ 在 459 和 $605 \mathrm{~cm}^{-1}$ 处只出现 了两个吸收峰, $459 \mathrm{~cm}^{-1}$ 处的峰归属于 $\mathrm{CeO}_{2}$ 立方萤 石结构的 $F_{2 \mathrm{~g}}$ 对称振动, ${ }^{20}$ 而 $605 \mathrm{~cm}^{-1}$ 处的峰归属为 氧空位不对称振动. ${ }^{21}$ 掺杂稀土后, 对应于 $\mathrm{CeO}_{2}$ 立方 茧石结构的 $F_{2 \mathrm{~g}}$ 对称振动的吸收峰发生移动, 这进一 步证明了稀土金属掺入 $\mathrm{CeO}_{2}$ 的晶格形成了固溶体 结构. 在相同检测条件下, 催化剂在 $605 \mathrm{~cm}^{-1}$ 处的吸 收峰和在 $459 \mathrm{~cm}^{-1}$ 处峰振动峰的强度比 $\left(A_{600} / A_{459}\right)$ 与

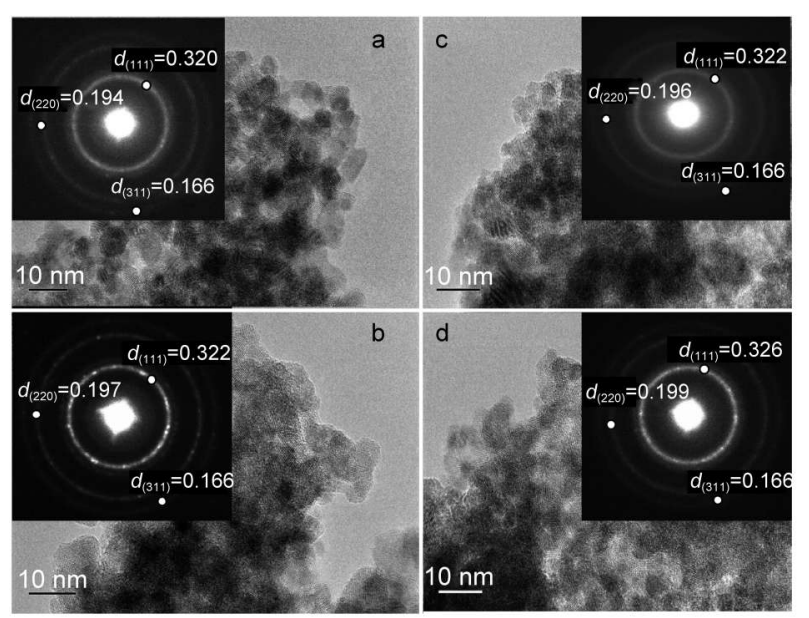

图 $3 \mathrm{CuO}-\mathrm{Ce}_{0.9} \mathrm{M}_{0.1} \mathrm{O}_{2}-\mathrm{SiO}_{2}$ 催化剂的 TEM 图及选区 电子衍射图(插图)

Fig.3 TEM images and selected area electron diffraction patterns (insets) of $\mathrm{CuO}-\mathrm{Ce}_{0.9} \mathrm{M}_{0.1} \mathrm{O}_{2}-\mathrm{SiO}_{2}$ catalysts (a) $\mathrm{CuO}-\mathrm{CeO}_{2}-\mathrm{SiO}_{2}$; (b) $\mathrm{CuO}-\mathrm{Ce}_{0.9} \mathrm{Pr}_{0.1} \mathrm{O}_{2}-\mathrm{SiO}_{2}$;

(c) $\mathrm{CuO}-\mathrm{Ce}_{0.9} \mathrm{Nd}_{0.1} \mathrm{O}_{2}-\mathrm{SiO}_{2}$; (d) $\mathrm{CuO}-\mathrm{Ce}_{0.9} \mathrm{La}_{0.1} \mathrm{O}_{2}-\mathrm{SiO}_{2}$. The unit of $d$ is nm. 


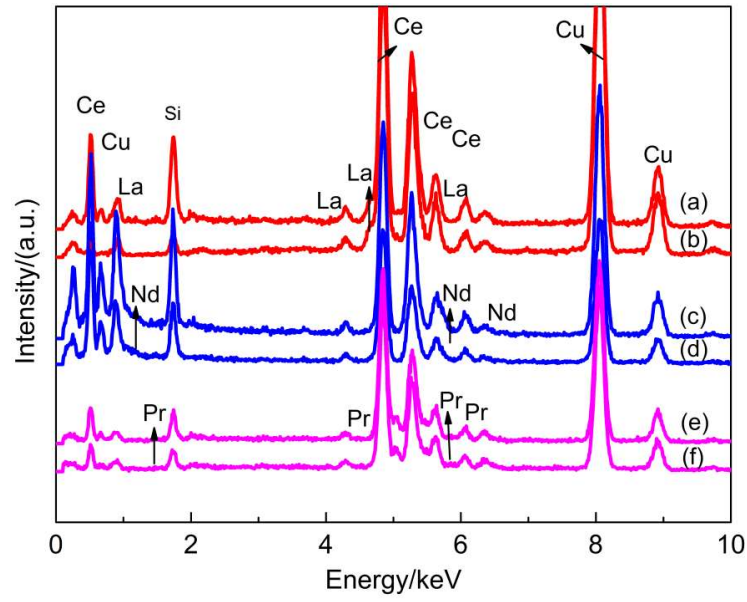

图 $4 \mathrm{CuO}-\mathrm{Ce}_{0.9} \mathrm{La}_{0.1} \mathrm{O}_{2}-\mathrm{SiO}_{2}(\mathrm{a}, \mathrm{b}), \mathrm{CuO}-\mathrm{Ce}_{0.9} \mathrm{Nd}_{0.1} \mathrm{O}_{2}-\mathrm{SiO}_{2}$ (c, d) 和 $\mathrm{CuO}-\mathrm{Ce}_{0.9} \mathrm{Pr}_{0.1} \mathrm{O}_{2}-\mathrm{SiO}_{2}(\mathrm{e}, \mathrm{f})$ 的能量散射 $\mathrm{X}$ 线 能谱(EDX)谱图

Fig.4 Energy dispersive X-ray spectroscopy (EDX) spectra of $\mathrm{CuO}-\mathrm{Ce}_{0.9} \mathrm{La}_{0.1} \mathrm{O}_{2}-\mathrm{SiO}_{2}(\mathrm{a}, \mathrm{b})$,

$\mathrm{CuO}-\mathrm{Ce}_{0.9} \mathrm{Nd}_{0.1} \mathrm{O}_{2}-\mathrm{SiO}_{2}(\mathrm{c}, \mathrm{d})$, and $\mathrm{CuO}-\mathrm{Ce}_{0.9} \mathrm{Pr}_{0.1} \mathrm{O}_{2}-\mathrm{SiO}_{2}(\mathrm{e}, \mathrm{f})$ $(a-f)$ corres ponding to the two different regions arbitrarily chosen from TEM images of $\mathrm{CuO}-\mathrm{Ce}_{0.9} \mathrm{M}_{0.1} \mathrm{O}_{2}-\mathrm{SiO}_{2}$ catalysts

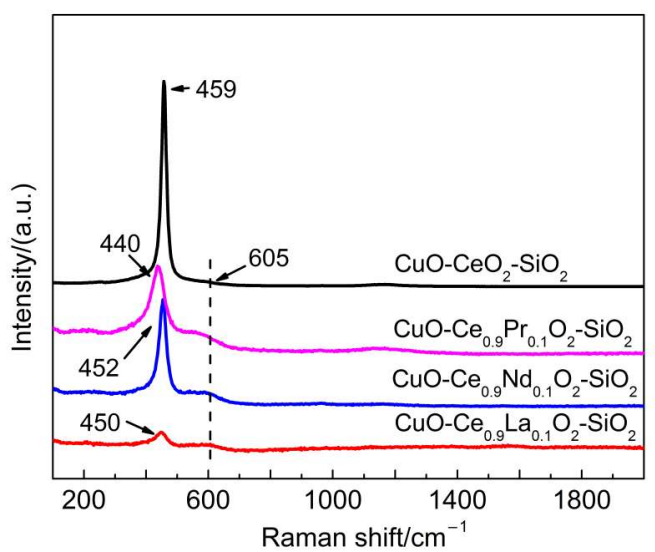

图 $5 \mathrm{CuO}-\mathrm{Ce}_{0.9} \mathrm{M}_{0.1} \mathrm{O}_{2}-\mathrm{SiO}_{2}$ 催化剂的 Raman 图谱

Fig.5 Raman spectroscopy of $\mathrm{CuO}-\mathrm{Ce}_{0.9} \mathrm{M}_{0.1} \mathrm{O}_{2}-\mathrm{SiO}_{2}$ catalysts

催化剂表面氧空位浓度成正比. ${ }^{22,23}$ 根据表 1 给出 $\mathrm{CuO}-\mathrm{Ce}_{0.9} \mathrm{M}_{0.1} \mathrm{O}_{2}-\mathrm{SiO}_{2}$ 催化剂的 $A_{605} / A_{459}$ 结果, 稀土的 掺杂可以显著提高固溶体的表面氧空位浓度, $\mathrm{CuO}-$ $\mathrm{Ce}_{0.9} \mathrm{La}_{0.1} \mathrm{O}_{2}-\mathrm{SiO}_{2}$ 氧空位浓度高达 $41.43 \%$.

\subsubsection{XPS 表征}

XPS 测试能够了解到催化剂表面组成和元素 价态等信息, 对 $\mathrm{CuO}-\mathrm{CeO}_{2}-\mathrm{SiO}_{2}$ 样品进行了 XPS 测 试. 催化剂表面 $\mathrm{Ce} 3 d$ 的光电子能谱见图 $6, \mathrm{Ce} 3 d$ 的 光电子能谱出现了八个特征峰. 其中 $u 、 u^{\prime \prime}$ 和 $u^{\prime \prime \prime}$ 为 $\mathrm{Ce}^{4+} 3 d_{3 / 2}$ 自旋-轨道多重谱线, $v 、 v^{\prime \prime}$ 和 $v^{\prime \prime \prime}$ 为 $\mathrm{Ce}^{4+} 3 d_{5 / 2}$ 自旋-轨道多重谱线, $u^{\prime}$ 和 $v^{\prime}$ 则分别为 $\mathrm{Ce}^{3+} 3 d_{3 / 2}$ 和

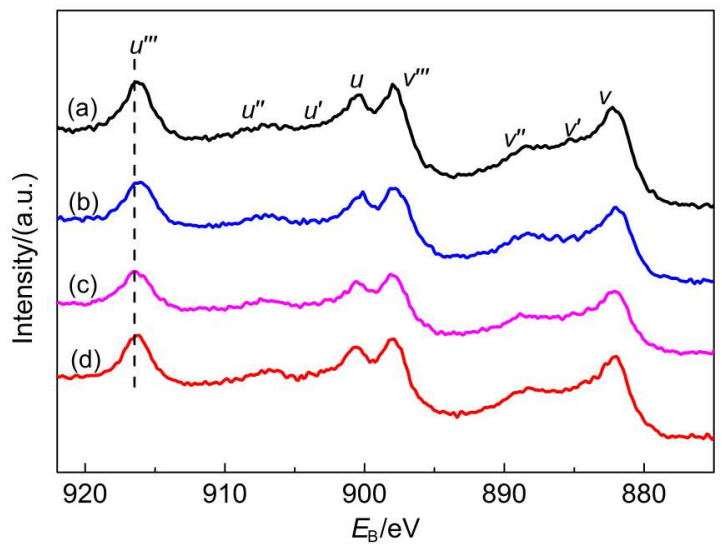

图 $6 \mathrm{CuO}-\mathrm{Ce}_{0.9} \mathrm{M}_{0.1} \mathrm{O}_{2}-\mathrm{SiO}_{2}$ 催化剂的 $\mathrm{Ce} 3 d \mathrm{XPS}$ 图谱

Fig.6 Ce $3 d$ XPS patterns of $\mathrm{CuO}-\mathrm{Ce}_{0.9} \mathrm{M}_{0.1} \mathrm{O}_{2}-\mathrm{SiO}_{2}$ catalysts

(a) $\mathrm{CuO}-\mathrm{CeO}_{2}-\mathrm{SiO}_{2}$; (b) $\mathrm{CuO}-\mathrm{Ce}_{0.9} \mathrm{Pr}_{0.1} \mathrm{O}_{2}-\mathrm{SiO}_{2}$; (c) $\mathrm{CuO}-\mathrm{Ce}_{0.9} \mathrm{Nd}_{0.1} \mathrm{O}_{2}-\mathrm{SiO}_{2}$; (d) $\mathrm{CuO}-\mathrm{Ce}_{0.9} \mathrm{La}_{0.1} \mathrm{O}_{2}-\mathrm{SiO}_{2}$

$\mathrm{Ce}^{3+} 3 d_{5 / 2}$ 的谱线. $\mathrm{Ce}^{3+}$ 相对含量可以由 $v^{\prime}$ 和 $u^{\prime}$ 拟合峰 面积与总峰面积估算得到(表 1). 可以看到, 稀土掺 杂后催化剂含有更高的 $\mathrm{Ce}^{3+}$ 浓度, 而 $\mathrm{Ce}^{3+}$ 浓度的提 高直接代表着催化剂表面形成了更多的氧空位和 不饱和化学键. 其中表面 $\mathrm{Ce}^{3+}$ 浓度顺序为: $\mathrm{CuO}$ $\mathrm{Ce}_{0.9} \mathrm{La}_{0.1} \mathrm{O}_{2}-\mathrm{SiO}_{2}>\mathrm{CuO}-\mathrm{Ce}_{0.9} \mathrm{Nd}_{0.1} \mathrm{O}_{2}-\mathrm{SiO}_{2}>\mathrm{CuO}-\mathrm{Ce}_{0.9} \mathrm{Pr}_{0.1} \mathrm{O}_{2-}$ $\mathrm{SiO}_{2}>\mathrm{CuO}-\mathrm{CeO}_{2}-\mathrm{SiO}_{2}$, 这与 Raman 测得的氧空位浓 度顺序是一致的. 另外, 催化剂的 $\operatorname{Pr} 3 d 、 \mathrm{Nd} 3 d$ 和 $\mathrm{La}$ $3 d$ 的 XPS 图谱如图 7 所示, Pr $3 d$ 在 933.8 和 953.0 $\mathrm{eV}$ 出现对应于 $\operatorname{Pr} 3 d_{5 / 2}$ 和 $\operatorname{Pr} 3 d_{3 / 2}$ 能级的峰, 这表明 $\mathrm{CuO}-\mathrm{Ce}_{0.9} \mathrm{Pr}_{0.1} \mathrm{O}_{2}-\mathrm{SiO}_{2}$ 催化剂中 $\mathrm{Pr}$ 元素存在 +3 和 +4 多种价态. ${ }^{24} \mathrm{CuO}-\mathrm{Nd}_{0.9} \mathrm{Pr}_{0.1} \mathrm{O}_{2}-\mathrm{SiO}_{2}$ 的 $\mathrm{Nd} 3 d$ 在 983.1 $\mathrm{eV}$ 出现对应于 $\mathrm{Nd} 3 d_{5 / 2}$ 的结合能峰, 而 $\mathrm{CuO}-$ $\mathrm{Ce}_{0.9} \mathrm{La}_{0.1} \mathrm{O}_{2}-\mathrm{SiO}_{2}$ 在 833.6、838.0、851.1 和 $854.8 \mathrm{eV}$ 可 以看到 $\mathrm{La} 3 d$ 的裂开的结合能的峰, 这与配位基 $(\mathrm{O}$ $2 p$ )至金属元素 (La 4f)之间的旋转轨道交互作用以 及电子转移有关, ${ }^{25}$ 这表明 $\mathrm{Nd}$ 和 $\mathrm{La}$ 主要是以单种氧 化价态形式存在. 样品的表面铜、稀土元素与表面 铈的含量比在表 1 给出. 表面稀土元素与表面铈含 量比和投入比例比较接近(10.0\%), 而各样品中表面 铜含量均远大于试剂投入比例(8.7\%), 显示铜物种 在表面富集, 表明铜物种主要分散在催化剂的表面.

\subsection{6 $\mathrm{H}_{2}$-TPR 结果}

$\mathrm{CuO}-\mathrm{Ce}_{0.9} \mathrm{M}_{0.1} \mathrm{O}_{2}-\mathrm{SiO}_{2}$ 催化剂的 $\mathrm{H}_{2}$ - TPR 谱图如 图 8 所示. $\mathrm{CuO}-\mathrm{CeO}_{2}-\mathrm{SiO}_{2}$ 催化剂在 $230{ }^{\circ} \mathrm{C}$ 左右出现 较强的还原峰, 可认为这主要归属于在固溶体表面 高分散的 $\mathrm{CuO}$ 的还原, ${ }^{15,26}$ 同时也可能含有少量固溶 体中 $\mathrm{Cu}^{2+}$ 的还原; ${ }^{27}$ 在 480 和 $700{ }^{\circ} \mathrm{C}$ 左右出现两个还 


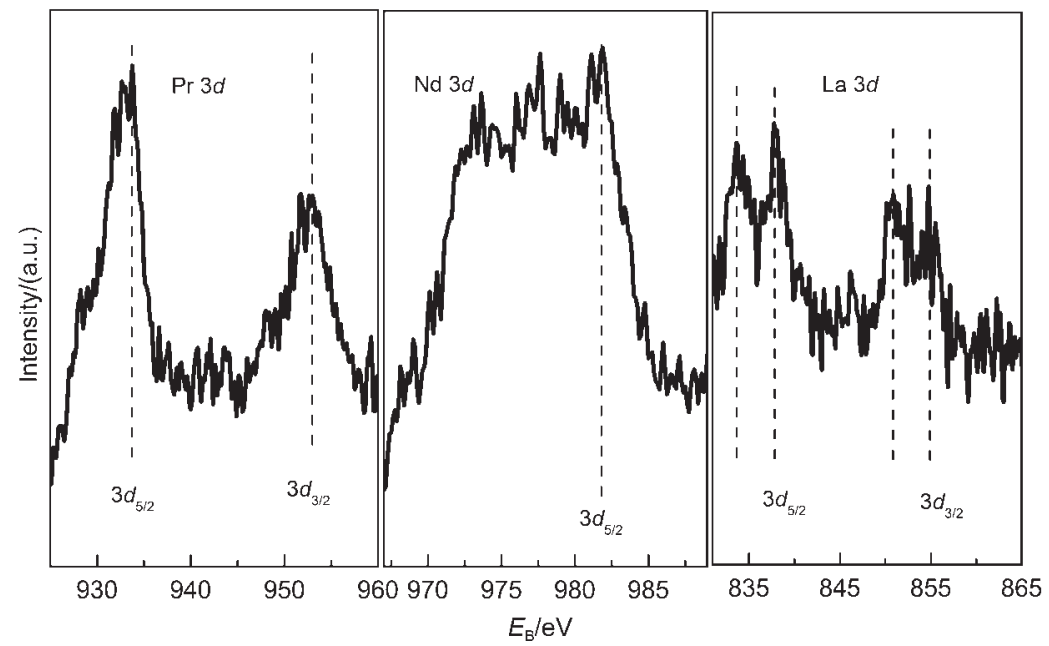

图 $7 \mathrm{CuO}-\mathrm{Ce}_{0.9} \mathrm{M}_{0.1} \mathrm{O}_{2}-\mathrm{SiO}_{2}$ 催化剂的 $\mathrm{Pr} 3 d, \mathrm{Nd} 3 d$ 和 $\mathrm{La} 3 d$ 的 XPS 图谱

Fig.7 Pr 3d, Nd 3d, and La $3 d$ XPS patterns of $\mathrm{CuO}-\mathrm{Ce}_{0.9} \mathrm{M}_{0.1} \mathrm{O}_{2}-\mathrm{SiO}_{2}$ catalysts

原峰, 这应归属于 $\mathrm{CeO}_{2}$ 的两步还原: 较低温度时发 生 $\mathrm{CeO}_{2}$ 表面氧物种的还原以及在较高温 $\mathrm{CeO}_{2}$ 体相 氧物种的还原. ${ }^{25} \mathrm{CuO}-\mathrm{Ce}_{0.9} \mathrm{M}_{0.1} \mathrm{O}_{2}-\mathrm{SiO}_{2}$ 催化剂对应于 铜物种的还原峰未出现明显的移动, 且除 $\mathrm{CuO}-$ $\mathrm{Ce}_{0.9} \mathrm{La}_{0.1} \mathrm{O}_{2}-\mathrm{SiO}_{2}$ 外其还原耗氢量未有明显的提升(表 1). 但是, 归属于 $\mathrm{CeO}_{2}$ 表面氧物种的还原峰强度有 所增加, 同时归属于 $\mathrm{CeO}_{2}$ 体相氧物种的还原峰均明 显向低温移动, 这可能与氧空位浓度的提高有关. 因为稀土掺入 $\mathrm{CeO}_{2}$ 的晶格中形成了固溶体结构并

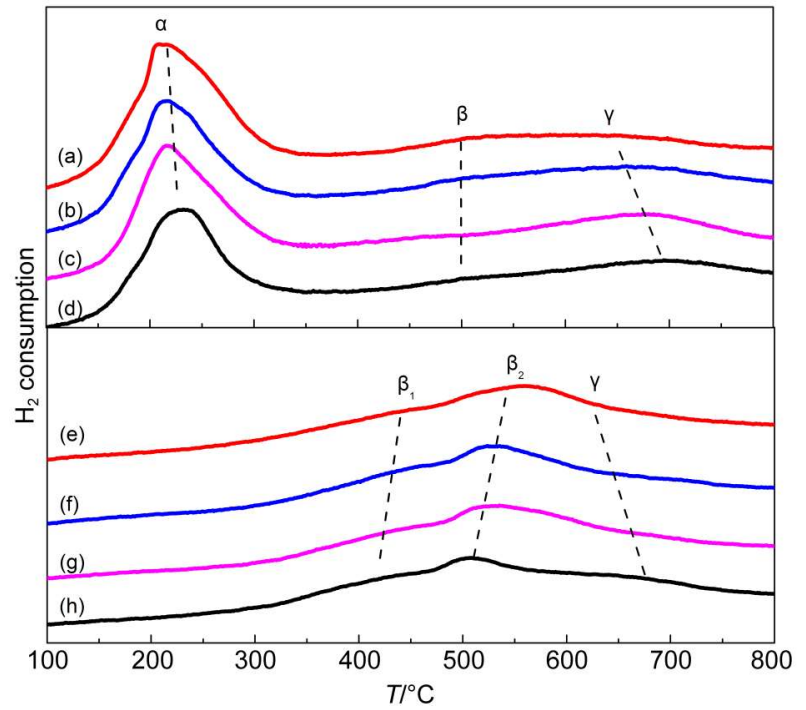

图 $8 \mathrm{CuO}-\mathrm{Ce}_{0.9} \mathrm{M}_{0.1} \mathrm{O}_{2}-\mathrm{SiO}_{2}$ 和 $\mathrm{Ce}_{0.9} \mathrm{M}_{0.1} \mathrm{O}_{2}-\mathrm{SiO}_{2}$ 催化剂的 $\mathrm{H}_{2}$-TPR 图

Fig.8 $\mathrm{H}_{2}$-TPR profiles of $\mathrm{CuO}-\mathrm{Ce}_{0.9} \mathrm{M}_{0.1} \mathrm{O}_{2}-\mathrm{SiO}_{2}$ and $\mathrm{Ce}_{0.9} \mathrm{M}_{0.1} \mathrm{O}_{2}-\mathrm{SiO}_{2}$ catalysts

(a) $\mathrm{CuO}-\mathrm{Ce}_{0.9} \mathrm{La}_{0.1} \mathrm{O}_{2}-\mathrm{SiO}_{2}$, (b) $\mathrm{CuO}-\mathrm{Ce}_{0.9} \mathrm{Nd}_{0.1} \mathrm{O}_{2}-\mathrm{SiO}_{2}$; (c) $\mathrm{CuO}-$ $\mathrm{Ce}_{0.9} \mathrm{Pr}_{0.1} \mathrm{O}_{2}-\mathrm{SiO}_{2}$; (d) $\mathrm{CuO}-\mathrm{CeO}_{2}-\mathrm{SiO}_{2}$; (e) $\mathrm{Ce}_{0.9} \mathrm{La}_{0.1} \mathrm{O}_{2}-\mathrm{SiO}_{2}$ (f) $\mathrm{Ce}_{0.9} \mathrm{Nd}_{0.1} \mathrm{O}_{2}-\mathrm{SiO}_{2} ;$ (g) $\mathrm{Ce}_{0.9} \mathrm{Pr}_{0.1} \mathrm{O}_{2}-\mathrm{SiO}_{2}$; (h) $\mathrm{CeO}_{2}-\mathrm{SiO}_{2}$
提高了氧空位浓度, 在一定程度上能够促进氧物种 在晶格中的流动, 这增强了表面氧和体相氧物种之 间的平衡, 使部分的体相氧物种能够在较低温度时 被还原. 总而言之, 稀土的掺杂未能明显改变铜物 种的氧化还原能力, 但固溶体的表面氧空位浓度却 影响了晶格中氧物种的氧化还原性能.

\section{2 $\mathrm{CuO}-\mathrm{Ce}_{0.9} \mathrm{M}_{0.1} \mathrm{O}_{2}-\mathrm{SiO}_{2}$ 催化剂的性能测试}

图 9 是 $\mathrm{CuO}-\mathrm{Ce}_{0.9} \mathrm{M}_{0.1} \mathrm{O}_{2}-\mathrm{SiO}_{2}$ 催化剂在不同温度 下活性测试结果. 可以看到, $\mathrm{CuO}-\mathrm{Ce}_{0} \mathrm{O}_{2}-\mathrm{SiO}_{2}$ 拥有良 好的催化活性, 且随温度的升高显著提高, $410^{\circ} \mathrm{C}$ 时, 氯化氢的转化率为 $55.0 \%$. 稀土掺杂后催化剂的 活性进一步提高, $\mathrm{CuO}-\mathrm{Ce}_{0.9} \mathrm{La}_{0.1} \mathrm{O}_{2}-\mathrm{SiO}_{2}$ 在 $410{ }^{\circ} \mathrm{C}$ 的 氯化氢转化率提升至 $66.8 \%$. 各催化剂的活性顺序 为: $\mathrm{CuO}-\mathrm{Ce}_{0.9} \mathrm{La}_{0.1} \mathrm{O}_{2}-\mathrm{SiO}_{2}>\mathrm{CuO}-\mathrm{Ce}_{0.9} \mathrm{Nd}_{0.1} \mathrm{O}_{2}-\mathrm{SiO}_{2}>$ $\mathrm{CuO}-\mathrm{Ce}_{0.9} \mathrm{Pr}_{0.1} \mathrm{O}_{2}-\mathrm{SiO}_{2}>\mathrm{CuO}-\mathrm{CeO}_{2}-\mathrm{SiO}_{2}$.

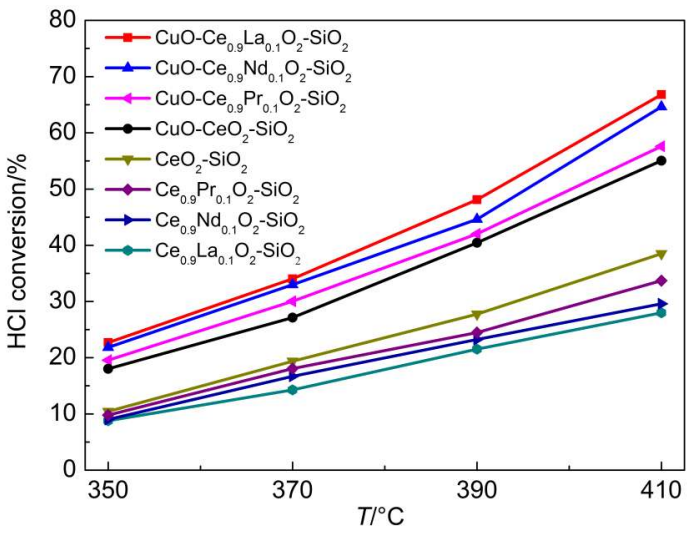

图9 不同反应温度下催化剂的活性

Fig.9 Catalytic activities of catalysts with different reaction temperatures 
结合 $\mathrm{CuO}-\mathrm{Ce}_{0.9} \mathrm{M}_{0.1} \mathrm{O}_{2}-\mathrm{SiO}_{2}$ 催化剂的表征结果, 稀土掺入 $\mathrm{CeO}_{2}$ 晶格中形成了固溶体结构, 获得更小 的晶粒尺寸和更大的比表面积, 并且显著增强了表 面氧空位浓度. 固溶体的结构(尤其表面氧空位的浓 度)显著影响了其氯化氢催化氧化活性. 表面氧空位 浓度越高的 $\mathrm{CuO}-\mathrm{Ce}_{0.9} \mathrm{La}_{0.1} \mathrm{O}_{2}-\mathrm{SiO}_{2}$ 催化剂, 其催化活 性也越高. 因此, 可认为固溶体的表面氧空位浓度 的提高是导致氯化氢催化氧化性能获得提升的关 键.

\section{3 $\mathrm{Ce}_{0.9} \mathrm{M}_{0.1} \mathrm{O}_{2}-\mathrm{SiO}_{2}$ 催化剂表征和性能测试}

对 $\mathrm{Ce}_{0.9} \mathrm{M}_{0.1} \mathrm{O}_{2}-\mathrm{SiO}_{2}(\mathrm{M}=\mathrm{La}, \mathrm{Pr}, \mathrm{Nd})$ 催化剂的结构 和性能的研究, 一定程度上能够有效地验证稀土掺 入所形成固溶体结构的差异, 是否为各催化剂性能 有所不同的关键. $\mathrm{Ce}_{0.9} \mathrm{M}_{0.1} \mathrm{O}_{2}-\mathrm{SiO}_{2}$ 催化剂的表征结果 列于表 2. 从表 2 可见, 掺杂稀土后, 催化剂的晶格常 数均变大, 这表明稀土进入了 $\mathrm{CeO}_{2}$ 晶格形成了固溶 体结构. 从 Scherrer 公式和 BET 法计算的平均晶粒 尺寸和比表面积结果看到, $\mathrm{Ce}_{0.9} \mathrm{M}_{0.1} \mathrm{O}_{2}-\mathrm{SiO}_{2}$ 可以获 得更小的晶粒尺寸和显著增大的比表面积. 此外, Raman 结果显示催化剂的表面氧空位浓度均显著提 高, 其中 $\mathrm{Ce}_{0.9} \mathrm{La}_{0.1} \mathrm{O}_{2}-\mathrm{SiO}_{2}$ 约为 $\mathrm{CeO}_{2}-\mathrm{SiO}_{2}$ 表面氧空位 的 3 倍. 从图 8 中的 $\mathrm{Ce}_{0.9} \mathrm{M}_{0.1} \mathrm{O}_{2}-\mathrm{SiO}_{2}$ 催化剂的 $\mathrm{H}_{2}-\mathrm{TPR}$ 结果看到, 在低温出现的两个还原峰 $\left(\beta_{1} 、 \beta_{2}\right)$ 均对应 于 $\mathrm{CeO}_{2}$ 表面氧物种的还原, 其峰强度明显增大且向 高温偏移, 而在高温出现对应于 $\mathrm{CeO}_{2}$ 体相氧物种的 还原峰 $(\gamma)$, 峰强度减小并明显向低温移动. 结合 $\mathrm{Ce}_{0.9} \mathrm{M}_{0.1} \mathrm{O}_{2}-\mathrm{SiO}_{2}$ 催化剂的 Raman 结果, 更高的氧空 位浓度能够增强表面氧和体相氧物种的之间的平 衡, 使更多的体相氧物种能够在较低温被还原. 对 比 $\mathrm{Ce}_{0.9} \mathrm{M}_{0.1} \mathrm{O}_{2}-\mathrm{SiO}_{2}$ 和 $\mathrm{CuO}-\mathrm{Ce}_{0.9} \mathrm{M}_{0.1} \mathrm{O}_{2}-\mathrm{SiO}_{2}$ 催化剂的 表征结果, 稀土掺杂均能形成良好的固溶体结构, 获得更小的晶粒尺寸和更大的比表面积, 且均不同 程度的增强了表面氧空位浓度以及表面氧和体相

表 $2 \mathrm{Ce}_{0.9} \mathrm{M}_{0.1} \mathrm{O}_{2}-\mathrm{SiO}_{2}$ 催化剂的物理化学性质

Table 2 Physical chemical properties of $\mathrm{Ce}_{0.9} \mathrm{M}_{0.1} \mathrm{O}_{2}-\mathrm{SiO}_{2}$ catalysts

\begin{tabular}{|c|c|c|c|c|}
\hline Sample & $\frac{\text { Cell parameter }}{\mathrm{nm}}$ & $\begin{array}{l}\text { Crystal } \\
\text { size } / \mathrm{nm}^{\mathrm{a}}\end{array}$ & $\frac{S_{\mathrm{BET}}}{\left(\mathrm{m}^{2} \cdot \mathrm{g}^{-1}\right)^{\mathrm{b}}}$ & $\frac{\left(A_{605} / A_{459}\right)}{\%^{\mathrm{c}}}$ \\
\hline $\mathrm{CeO}_{2}-\mathrm{SiO}_{2}$ & 0.5401 & 5.0 & 149.4 & 5.54 \\
\hline $\mathrm{Ce}_{0.9} \mathrm{Pr}_{0.1} \mathrm{O}_{2}-\mathrm{SiO}_{2}$ & 0.5412 & 4.7 & 170.0 & 12.09 \\
\hline $\mathrm{Ce}_{0.9} \mathrm{Nd}_{0.1} \mathrm{O}_{2}-\mathrm{SiO}_{2}$ & 0.5418 & 4.4 & 156.2 & 14.86 \\
\hline $\mathrm{Ce}_{0.9} \mathrm{La}_{0.1} \mathrm{O}_{2}-\mathrm{SiO}_{2}$ & 0.5424 & 4.2 & 159.0 & 17.35 \\
\hline
\end{tabular}

${ }^{\mathrm{a}}$ calculation from the peak during $28.4^{\circ}$ in the XRD pattern using the Scherrer equation, ${ }^{\mathrm{b}}$ surface area derived from BET method, ${ }^{\circ} A_{596} / A_{461}$ calculated from Raman results
氧物种之间的平衡.

$\mathrm{Ce}_{0.9} \mathrm{M}_{0.1} \mathrm{O}_{2}-\mathrm{SiO}_{2}$ 催化剂在不同温度下活性测试 如图 9 所示. $\mathrm{CeO}_{2}-\mathrm{SiO}_{2}$ 具备一定的催化活性, $410{ }^{\circ} \mathrm{C}$ 时氯化氢的转化率为 $38.5 \%$. 稀土掺杂后催化剂的 活性却不同程度地降低, 各催化剂的活性顺序为: $\mathrm{Ce}_{0.9} \mathrm{La}_{0.1} \mathrm{O}_{2}-\mathrm{SiO}_{2}<\mathrm{Ce}_{0.9} \mathrm{Nd}_{0.1} \mathrm{O}_{2}-\mathrm{SiO}_{2}<\mathrm{Ce}_{0.9} \mathrm{Pr}_{0.1} \mathrm{O}_{2}-\mathrm{SiO}_{2}<$ $\mathrm{CeO}_{2}-\mathrm{SiO}_{2}$. 对比 $\mathrm{Ce}_{0.9} \mathrm{M}_{0.1} \mathrm{O}_{2}-\mathrm{SiO}_{2}$ 和 $\mathrm{CuO}-\mathrm{Ce}_{0.9} \mathrm{M}_{0.1} \mathrm{O}_{2}-$ $\mathrm{SiO}_{2}$ 催化剂的性能结果, 稀土掺杂后, $\mathrm{CuO}-$ $\mathrm{Ce}_{0.9} \mathrm{M}_{0.1} \mathrm{O}_{2}-\mathrm{SiO}_{2}$ 催化活性显著提高, $\mathrm{Ce}_{0.9} \mathrm{M}_{0.1} \mathrm{O}_{2}-\mathrm{SiO}_{2}$ 催化活性却显著降低. 另外, 对于 $\mathrm{CuO}-\mathrm{Ce}_{0.9} \mathrm{M}_{0.1} \mathrm{O}_{2}-$ $\mathrm{SiO}_{2}$, 氧空位浓度越高其催化活性越高, 而对于 $\mathrm{Ce}_{0.9} \mathrm{M}_{0.1} \mathrm{O}_{2}-\mathrm{SiO}_{2}$, 较高的氧空位浓度却导致了更低的 催化活性. 显然, 稀土掺杂导致了氧空位浓度的增 加以及表面氧和体相氧物种之间的平衡的增强, 并 不能直接增强固溶体表面发生的氯化氢氧化反应.

\section{4 反应级数测试}

为了更加深入了解稀土掺杂对催化剂的氯化 氢氧化表面反应过程的影响, 对 $\mathrm{CuO}-\mathrm{CeO}_{2}-\mathrm{SiO}_{2}$, $\mathrm{CuO}-\mathrm{Ce}_{0.9} \mathrm{La}_{0.1} \mathrm{O}_{2}-\mathrm{SiO}_{2}, \mathrm{CeO}_{2}-\mathrm{SiO}_{2}$ 和 $\mathrm{Ce}_{0.9} \mathrm{La}_{0.1} \mathrm{O}_{2}-\mathrm{SiO}_{2}$ 四个催化剂的反应级数进行了测定. 由于 $\mathrm{HCl}$ 催化 氧化反应为可逆反应, 反应速率的幂函数方程可以 表达为:

$$
r_{\mathrm{HCl}}=k p_{\mathrm{O}_{2}}^{\alpha} p_{\mathrm{HCl}}^{\beta}-k^{\prime} p_{\mathrm{Cl}_{2}}^{\gamma} p_{\mathrm{H}_{2} \mathrm{O}}^{\sigma}
$$

式中, $p_{\mathrm{O}_{2}}$ 和 $p_{\mathrm{HCl}}$ 分别为反应物 $\mathrm{O}_{2}$ 和 $\mathrm{HCl}$ 的分压, $p_{\mathrm{Cl}_{2}}$ 和 $p_{\mathrm{H}_{2} \mathrm{O}}$ 则分别为逆反应时 $\mathrm{Cl}_{2}$ 和 $\mathrm{H}_{2} \mathrm{O}$ 的分压. 其中 $\alpha 、 \beta 、 \gamma$ 和 $\delta$ 分别为催化剂对 $\mathrm{O}_{2} 、 \mathrm{HCl} 、 \mathrm{Cl}_{2}$ 和 $\mathrm{H}_{2} \mathrm{O}$ 的反应级数. 在催化剂动力学测试过程中将转化率限制在 5\%$20 \%$, 故反应式中 $k p_{\mathrm{O}_{2}}^{\alpha} p_{\mathrm{HCl}}^{\beta}>k^{\prime} p_{\mathrm{C}_{2}}^{\nu} p_{\mathrm{H}_{2} \mathrm{O}}^{\sigma}$, 因此这里忽 略逆反应的影响并简化方程如下:

$$
r_{\mathrm{HCl}}=k p_{\mathrm{O}_{2}}^{\alpha} p_{\mathrm{HCl}}^{\beta}
$$

反应级数 $\alpha, \beta$ 可采用微分法测得. 测试时以 $\mathrm{N}_{2}$ 为平 衡气, 保持 $\mathrm{HCl}$ 的分压 $\left(p_{\mathrm{HCl}}\right)$ 不变, 测得不同 $p_{\mathrm{O}_{2}}$ 下 $r_{\mathrm{HCl}}$ 值, 以 $\ln p_{\mathrm{O}_{2}}$ 对 $\ln r_{\mathrm{HC}}$ 作图, 斜率即为 $\alpha$, 同理可测得 $\beta$.

各催化剂对 $\mathrm{O}_{2}$ 和 $\mathrm{HCl}$ 的反应级数测试结果列

\section{表3 各催化剂对 $\mathrm{O}_{2}$ 和 $\mathrm{HCl}$ 的反应级数}

\section{Table 3 Reaction orders of $\mathrm{O}_{2}$ and $\mathrm{HCl}$ of the catalysts}

\begin{tabular}{lcc}
\hline \multicolumn{1}{c}{ Catalyst } & $\alpha\left(\mathrm{O}_{2}\right)^{\mathrm{a}}$ & $\beta(\mathrm{HCl})^{\mathrm{b}}$ \\
\hline $\mathrm{CeO}_{2}-\mathrm{SiO}_{2}$ & 0.50 & 0.42 \\
$\mathrm{CuO}-\mathrm{CeO}_{2}-\mathrm{SiO}_{2}$ & 0.46 & 0.73 \\
$\mathrm{Ce}_{0.9} \mathrm{La}_{0.1} \mathrm{O}_{2}-\mathrm{SiO}_{2}$ & 0.77 & 0.10 \\
$\mathrm{CuO}-\mathrm{Ce}_{0.9} \mathrm{La}_{0.1} \mathrm{O}_{2}-\mathrm{SiO}_{2}$ & 0.65 & 0.15 \\
\hline
\end{tabular}

${ }^{a}$ the reaction rate of $\mathrm{O}_{2}$ over the catalysts, ${ }^{b}$ the reaction rate of $\mathrm{HCl}$ over the catalysts 
于表 3. 可以看到, $\mathrm{CeO}_{2}-\mathrm{SiO}_{2}$ 对 $\mathrm{O}_{2}$ 和 $\mathrm{HCl}$ 的反应级 数分别为 0.50 和 0.42 , 这表明 $\mathrm{O}_{2}$ 分压和 $\mathrm{HCl}$ 分压均 显著影响氯化氢氧化反应速率. 在反应过程中, 生 成的 $\mathrm{Cl}_{2}$ 首先会吸附于催化剂的表面, 而 $\mathrm{O}_{2}$ 和 $\mathrm{HCl}$ 作为反应物将会与生成的 $\mathrm{Cl}_{2}$ 在催化剂表面产生竞 争吸附, 因此更高的 $\mathrm{O}_{2}$ 分压以及 $\mathrm{HCl}$ 分压均会加快 $\mathrm{Cl}_{2}$ 的脱附, 从而提高 $\mathrm{Cl}_{2}$ 产率. 可依此推断, 在此过 程中 $\mathrm{Cl}_{2}$ 的脱附是这个反应过程的决速步骤. $\mathrm{CuO}-$ $\mathrm{CeO}_{2}-\mathrm{SiO}_{2}$ 对 $\mathrm{O}_{2}$ 和 $\mathrm{HCl}$ 的反应级数也较大, 分别为 0.46 和 0.73 , 表明 $\mathrm{Cl}_{2}$ 的脱附仍为反应过程的决速步 骤. 但是 $\mathrm{CuO}-\mathrm{CeO}_{2}-\mathrm{SiO}_{2}$ 对 $\mathrm{HCl}$ 的反应级数明显更 大, 此时 $\mathrm{HCl}$ 的分压对氯化氢氧化反应速率的影响 更加显著, 这主要因为 $\mathrm{CuO}$ 的存在能够提供更多的 活性点位用来吸附和活化 $\mathrm{HCl}$.

稀土掺杂后, $\mathrm{Ce}_{0.9} \mathrm{La}_{0.1} \mathrm{O}_{2}-\mathrm{SiO}_{2}$ 和 $\mathrm{CuO}-\mathrm{Ce}_{0.9} \mathrm{La}_{0.1} \mathrm{O}_{2}$ $\mathrm{SiO}_{2}$ 对 $\mathrm{O}_{2}$ 的反应级数分别增大到 0.77 和 0.65 , 而对 $\mathrm{HCl}$ 的反应级数均显著较小至 0.10 和 0.15 , 这说明 $\mathrm{O}_{2}$ 分压对氯化氢氧化反应速率的影响远大于 $\mathrm{HCl}$ 分 压, 此时氧分子的吸附成为这个反应过程的决速步 骤.

\section{5 机理分析}

根据前期的研究工作, ${ }^{15} \mathrm{CuO}-\mathrm{Ce}_{0.9} \mathrm{M}_{0.1} \mathrm{O}_{2}-\mathrm{SiO}_{2}$ 催 化剂上氯化氢氧化表面反应过程可用图 10 表示. $\mathrm{HCl}$ 主要通过以下三种途径发生氧化反应: (1) 这一 过程主要遵循 Langmuir-Hinshelwood 机理, ${ }^{28} \mathrm{HCl}$ 和 $\mathrm{O}_{2}$ 均吸附于表面高分散的 $\mathrm{CuO}$ 上并发生反应. (2) 这一个过程比较复杂, 可以用 Mar-van-Krevelen 机 理进行描述: 一分子 $\mathrm{HCl}$ 首先与固溶体的晶格氧发 生反应生成 $-\mathrm{OH}, \mathrm{Cl}$ 原子会占据氧空位, 另一分子 $\mathrm{HCl}$ 与 $-\mathrm{OH}$ 反应生成 $\mathrm{H}_{2} \mathrm{O}$, 生成 $\mathrm{H}_{2} \mathrm{O}$ 脱附后留下的 氧空位被 $\mathrm{Cl}$ 原子占据, 占据氧空位的 $\mathrm{Cl}$ 原子活化到 固溶体的表面并生成 $\mathrm{Cl}_{2}$, 而 $\mathrm{O}_{2}$ 是通过氧空位进行 吸附和活化. ${ }^{11,29}$ (3) 这一过程可以被认为是对途径

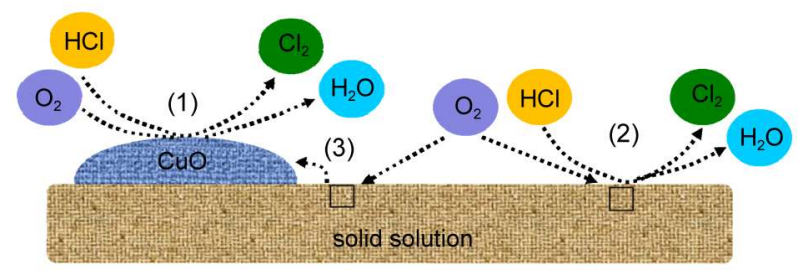

图 $10 \mathrm{CuO}-\mathrm{Ce}_{0.9} \mathrm{M}_{0.1} \mathrm{O}_{2}-\mathrm{SiO}_{2}$ 催化剂上氯化氢 氧化表面反应过程图

Fig.10 Possible reaction pathways of $\mathrm{HCl}$ oxidation on the surface of $\mathrm{CuO}-\mathrm{Ce}_{0.9} \mathrm{M}_{0.1} \mathrm{O}_{2}-\mathrm{SiO}_{2}$ catalyst
(1)的补充, $\mathrm{O}_{2}$ 通过氧空位实现氧的吸附, 并存在“氧 溢流”现象: 氧物种从固溶体的溢流至在其表面高 分散 $\mathrm{CuO}$ 上, 并与在高分散 $\mathrm{CuO}$ 上吸附的 $\mathrm{HCl}$ 发生 反应. 对于 $\mathrm{CuO}-\mathrm{CeO}_{2}-\mathrm{SiO}_{2}$ 催化剂, 其表面发生的反 应主要是通过途径(1)和(3)上进行的.

对于 $\mathrm{CeO}_{2}-\mathrm{SiO}_{2}$ 催化剂, 其表面反应过程为途径 (2). 稀土改性后, 氧空位浓度显著增强但催化活性 却有所下降. 根据密度泛函理论计算研究结果, 稀 土掺杂后导致固溶体晶胞扩张, 使较低的 $\mathrm{O}_{2}$ 分压下 $\left(V\left(\mathrm{O}_{2}\right): V(\mathrm{HCl})=1\right)$, 占据氧空位的 $\mathrm{Cl}$ 原子更难被 $\mathrm{O}$ 所 取代并进行脱附, 从而导致了更低的反应速率. ${ }^{17}$ 反 应级数结果表明 $\mathrm{CuO}-\mathrm{Ce}_{0.9} \mathrm{M}_{0.1} \mathrm{O}_{2}-\mathrm{SiO}_{2}$ 与 $\mathrm{Ce}_{0.9} \mathrm{M}_{0.1} \mathrm{O}_{2}-$ $\mathrm{SiO}_{2}$ 催化剂一样, 分子氧从催化剂氧空位的的吸附 和活化为其决速步骤, 此时途径(2)的反应速率同样 会降低. 然而, 此时催化剂表面的反应还存在途径 (1)和(3). 稀土掺杂增强了固溶体的表面氧空位浓 度, 使固溶体表面氧空位吸附的氧物种快速溢流至 表面高分散 $\mathrm{CuO}$ 上, 并与在 $\mathrm{CuO}$ 上吸附的 $\mathrm{HCl}$ 发生 反应. 可见, 稀土掺杂后固溶体表面更高的氧空位 浓度增强了固溶体表面的“氧溢流”, 使进行途径(3) 的比重更大, 加快了催化剂氯化氢氧化的整体反应 速率, 这是 $\mathrm{CuO}-\mathrm{Ce}_{0.9} \mathrm{M}_{0.1} \mathrm{O}_{2}-\mathrm{SiO}_{2}$ 具备更高活性的关 键.

\section{4 结 论}

采用模板法制备了 $\mathrm{CuO}-\mathrm{CeO}_{2}-\mathrm{SiO}_{2}$ 以及稀土掺 杂的 $\mathrm{CuO}-\mathrm{Ce}_{0.9} \mathrm{M}_{0.1} \mathrm{O}_{2}-\mathrm{SiO}_{2}$ 催化剂. 表征结果显示稀 土掺杂进入 $\mathrm{CeO}_{2}$ 形成良好的固溶体结构, 导致更小 的晶粒尺寸和更高的比表面积以及显著增强的表 面氧空位浓度. 性能测试结果显示, 稀土掺杂的 $\mathrm{CuO}-\mathrm{Ce}_{0.9} \mathrm{M}_{0.1} \mathrm{O}_{2}-\mathrm{SiO}_{2}$ 催化剂的氯化氢氧化反应活性 均显著提升, 且固溶体氧空位浓度的高低与氯化氢 氧化活性直接相关. 通过与 $\mathrm{Ce}_{0.9} \mathrm{M}_{0.1} \mathrm{O}_{2}-\mathrm{SiO}_{2}$ 催化剂 的结构和性能的对比, 发现氧空位浓度的提高不能 导致固溶体表面发生的氯化氢氧化反应活性的提 升. 稀土掺杂后分子氧的吸附成为反应过程的决速 步骤, 但在 $V\left(\mathrm{O}_{2}\right): V(\mathrm{HCl})=1$ 条件下, 更高的氧空位浓 度反而降低了固溶体表面的反应速率. 机理分析认 为, 对于 $\mathrm{CuO}-\mathrm{Ce}_{0.9} \mathrm{M}_{0.1} \mathrm{O}_{2}-\mathrm{SiO}_{2}$, 稀土掺杂提高了固溶 体的表面氧空位, 这能够增强固溶体表面的“氧溢 流”: 氧空位吸附的氧物种快速溢流至表面高分散 $\mathrm{CuO}$ 上, 并与在 $\mathrm{CuO}$ 上吸附的 $\mathrm{HCl}$ 发生反应, 加快 了催化剂氯化氢氧化的整体反应速率. 这是 $\mathrm{CuO}$ - 
$\mathrm{Ce}_{0.9} \mathrm{M}_{0.1} \mathrm{O}_{2}-\mathrm{SiO}_{2}$ 具备高活性的关键.

\section{References}

(1) Pérez-Ramírez, J.; Mondelli, C.; Schmidt, T.; Schlüter, O. F. K.; Wolf, A.; Mleczko, L.; Dreier, T. Energy Environ. Sci. 2011, 4, 4786. doi: 10.1039/c1ee02190g

(2) Deacon, H. Manufacture of Chlorine. U. S. Pat. 85370A, 1868.

(3) Crihan, D.; Knapp, M.; Zweidinger, S.; Lundgren, E.; Weststrate, C. J.; Andersen, J. N.; Seitsonen A. P.; Over, H. Angew. Chem. Int. Edit. 2008, 47, 2131.

(4) Tang, J. H.; Chen, X.; Fei, Z. Y.; Zhao, J. H.; Cui, M. F.; Qiao, X. Ind. Eng. Chem. Res. 2013, 52, 11897. doi: 10.1021/ ie $400200 \mathrm{~g}$

(5) Seki, K. Catal. Surv. Asia 2010, 14, 168. doi: 10.1007/s10563010-9091-7

(6) Mondelli, C.; Amrute, A. P.; Krumeich, F.; Schmidit, T.; PérezRamírez, J. ChemCatChem 2011, 3, 657. doi: 10.1002/ cctc. 201000424

(7) Mondelli, C.; Amrute, A. P.; Schmidt, T.; Pérez-Ramírez, J. Chem. Commun. 2011, 47, 7173.

(8) Hernandez, W. Y.; Laguna, O. H.; Centeno, M. A.; Odriozola, J. A. J. Solid State Chem. 2011, 184, 3014. doi: 10.1016/j. jssc.2011.09.018

(9) Cao, H. Y.; Wang, J. L.; Yan, S. H.; Liu, Z. M.; Gong, M. C.; Chen, Y. Q. Acta. Phys. -Chim. Sin. 2012, 28 (8), 1936. [曹红 岩, 王健礼, 间生辉, 刘志敏, 龚茂初, 陈耀强. 物理化学学报, 2012, 28 (8), 1936.] doi: 10.3866/PKU.WHXB201205173

(10) Wang, S. Y.; Li, N.; Luo, L. F.; Huang, W. X.; Pu, Z. Y.; Wang, J. W.; Hu, G. S.; Luo, M. F.; Lu, J. Q. Appl. Catal. B: Environ. 2014, 144, 325 .

(11) Amrute, A. P.; Mondelli, C.; Moser, M.; Novell-Leruth, G.; López, N.; Rosenthal, D.; Farra, R.; Schüster, M. E.; Teschner, D.; Schmidt, T.; Pérez-Ramírez, J. J. Catal. 2012, 286, 287. doi: 10.1016/j.jcat.2011.11.016

(12) Moser, M.; Mondelli, C.; Schmidt, T.; Girgsdies, F.; Schüster, M. E.; Farra, R.; Szentmiklósi, L.; Teschner, D.; Pérez-Ramírez, J. Appl. Catal. B: Environ. 2013, 132-133, 123.

(13) Amrute, A. P.; Larrazábal, G. O.; Mondelli, C.; Pérez Ramírez, J. Angew. Chem. Int. Edit. 2013, 52, 9772. doi: 10.1002/ ange.201304254

(14) Chen, X.; Lü, G. M.; Tang, J. H.; Cui, M. F.; Zhou, Z.; Cao, R.; Qiao, X. J. Chem. Eng. Chin. Univ. 2011, 25, 109. [陈献, 吕 高明, 汤吉海, 崔咪芬, 周 哲, 曹 锐, 乔 旭. 高校化学工程
学报, 2011, 25, 109.]

(15) Fei, Z. Y.; Liu, H. Y.; Dai, Y.; Ji, W. J.; Chen, X.; Tang, J. H.; Cui, M. F.; Qiao, X. Chem. Eng. J. 2014, 257, 273. doi: 10.1016/ j.cej.2014.07.033

(16) Jampaiah, D.; Tur, K. M.; Ippolito, S. J.; Sabri, Y. M.; Tardio, J.; Bhargava, S. K.; Reddy, B. M. RSC. Adv. 2013, 3, 12963. doi: $10.1039 / \mathrm{c} 3 \mathrm{ra} 41441 \mathrm{~h}$

(17) Farra, R.; García-Melchor, M.; Eichelbaum, M.; Hashagen, M.; Frandsen, W.; Allan, J.; Girgsdies, F.; Szentmiklósi, L.; López, N.; Teschner, D. ACS Catal. 2013, 3, 2256. doi: 10.1021/ cs4005002

(18) Jiang, J. T.; Wei, X. J.; Xu, C. Y.; Zhou, Z. X.; Zhen, L. J. Magn. Magn. Mater. 2013, 334, 111. doi: 10.1016/j.jmmm.2012.12.036

(19) Hernandez, W. Y.; Laguna, O. H.; Centeno, M. A.; Odriozola, J. A. J. Solid State Chem. 2011, 184, 3014. doi: 10.1016/j. jssc.2011.09.018

(20) Si, R.; Zhang, Y. W.; Li, S. J.; Lin, B. X.; Yan, C. H. J. Phys. Chem. B 2004, 33, 12481.

(21) Meng, Z. H.; Yang, P.; Zhou, R. X. Acta Phys. -Chim. Sin. 2013 29 (2), 391. [孟中华, 杨 鹏, 周仁贤. 物理化学学报, 2013, 29 (2), 391.] doi: 10.3866/PKU.WHXB201212072

(22) Yang, D.; Wang, L.; Sun, Y. Z.; Zhou, K. J. Phys. Chem. C 2010, 114, 8926. doi: 10.1021/jp912227p

(23) Liu, L.; Yao, Z.; Deng, Y.; Gao, F.; Liu, B.; Dong, L. ChemCatChem 2011, 3, 978. doi: 10.1002/cctc.v3.6

(24) Reddy, B. M.; Saikia, P.; Bharali, P.; Park, S. E.; Muhler, M.; Grüunert, W. J. Phys. Chem. C 2009, 113, 2452. doi: 10.1021/ jp809837g

(25) Katta, L.; Sudarsanam, P.; Thrimurthulu, G.; Reddy, B. M. Appl. Catal. B: Environ. 2010, 101, 101. doi: 10.1016/j. apcatb.2010.09.012

(26) Gao, X.; Du, X. S.; Cui, L. W.; Fu, Y. C.; Luo, Z. Y.; Cen, K. F. Catal. Commun. 2010, 12, 255.

(27) Menon, U.; Poelman, H.; Bliznuk, V.; Galvita, V. V.; Poelman, D.; Marin, G. B. J. Catal. 2012, 295, 91. doi: 10.1016/j. jcat.2012.07.026

(28) Amrute, A. P.; Mondelli, C.; Miguel, A. G.; Hevia, P. J. J. Phys. Chem. C 2011, 115, 1056

(29) Farra, R.; Wrabetz, S.; Schuster, E. S.; Stotz, E.; Hamilton, N. G.; Amrute, A. P.; Pérez-Ramírez, J.; López, N.; Teschner, D. Phys. Chem. Chem. Phys. 2013, 15, 3454. doi: 10.1039/ c2cp $42767 \mathrm{~b}$ 